\title{
Modeling the pinning of Au and Ni clusters on graphite
}

\author{
R. Smith, C. Nock, and S. D. Kenny \\ Department of Mathematical Sciences, Loughborough University, Loughborough, Leicestershire LE11 3TU, United Kingdom
}

Joseph J. Belbruno

Department of Chemistry, Dartmouth College, Hanover, New Hampshire, USA

M. Di Vece, S. Palomba, and R. E. Palmer

Nanoscale Physics Research Laboratory, School of Physics and Astronomy, The University of Birmingham, Edgbaston, Birmingham, B15 2TT, United Kingdom

(Received 19 October 2005; published 27 March 2006)

\begin{abstract}
The pinning of size-selected $\mathrm{Au}_{N}$ and $\mathrm{Ni}_{N}$ clusters on graphite, for $N=7-100$, is investigated by means of molecular dynamics simulations and the results are compared to experiment and previous work with $\mathrm{Ag}$ clusters. Ab initio calculations of the binding of the metal adatom and dimers on a graphite surface are used to parametrize the potentials used in the simulations. The clusters are projected normally towards a graphite surface and the value of the energy at which pinning first occurs, $E_{P}$, is determined. Pinning is shown to occur when a surface defect, made by the cluster interaction, is first produced. The simulations give a good agreement with the experimentally determined pinning energy thresholds and the heights of the clusters on the surface. The gold clusters are shown to be flatter and more spread out than the nickel clusters which are more compact.
\end{abstract}

DOI: 10.1103/PhysRevB.73.125429

\section{INTRODUCTION}

Size-selected nanoclusters have attracted considerable interest recently because of the potential application of dispersed arrays of these clusters on surfaces. For example, gold clusters form sites at which proteins or other organic molecules can be immobilized, ${ }^{1,2}$ leading to the intriguing possibility of nanoscale biosensors. If the nanoclusters are projected towards the surface with a certain energy, then different energy deposition regimes result in different interaction processes between the cluster and the substrate. Depending on the energy, the clusters can either soft-land and diffuse, pin on the surface, or implant beneath. Previous work using Ag clusters on graphite has shown that the clusters can pin on the surface if a certain energy threshold $E_{P}$ is surpassed. ${ }^{3-5}$ It was also found that the pinning threshold for $\mathrm{Ag}$ clusters had a linear variation with $N$, the number of atoms in the cluster. Below $E_{P}$ the clusters diffuse across the surface with high mobility and aggregate at defects. If the energy is increased further above $E_{P}$ then the clusters implant below the surface with an implantation depth that varies linearly with cluster velocity and inversely with cluster cross-sectional area $\left(N^{2 / 3}\right) \cdot{ }^{6-8}$

Other cluster species that have potentially interesting applications include gold, because of its applications with proteins or in electronics and catalysis, and nickel which has also similar applications and additionally has magnetic properties. These materials have been the subject of experimental investigation via the deposition of such size-selected clusters on surfaces experimentally. ${ }^{2-4,6,7,9,10}$ Recently a wide ranging experimental study of the pinning thresholds for gold and nickel clusters has been made for $N$ ranging from 10 to 300 . Here we investigate the deposition of size-selected Au and Ni clusters using molecular dynamics computer simulations.

Previous experimental and theoretical work with Ag clusters determined that $E_{P}$ was the value at which the impacting
PACS number(s): 79.20.Rf, 61.46.-w, 81.07.-b, 68.55.Ln

cluster had sufficient energy permanently to displace a surface carbon atom from its $s p^{2}$ bonding site configuration to create a surface defect to which the cluster could bond. The purpose of this investigation is to perform a series of molecular dynamics simulations for both $\mathrm{Au}$ and Ni clusters of different sizes to compare with experimental results and to investigate if the physical mechanisms for pinning agree with the previous conclusions for Ag.

\section{GOLD AND SILVER ADATOMS AND DIMERS ON GRAPHITE}

In this section the bonding of $\mathrm{Ag}$ and $\mathrm{Au}$ adatoms and dimers to graphite is considered using ab initio calculations so that parameters for a classical potential energy function can be determined. To perform these calculations the $a b$ initio, density functional theory code PLATO was used. ${ }^{11}$ The main details of the work are reported in Refs. 12 and 13 and here only the relevant parts necessary for the parametrization are described.

The energy barriers have not been specifically calculated but we have calculated the binding energy of the adatoms at various points along the path between $\alpha$ and $\beta$ sites along the $\langle 110\rangle$ direction. The path is shown in Fig. 1 together with the layout of atoms in the surface. As can be seen in Table I, the energy difference between the points along the path is very small. Such low barriers are an indication that diffusion of the adatoms over the surface would readily occur.

Results in Table I show that the $\beta$ sites, shown in Fig. 1, are slightly preferred for single atoms, but the differences in binding energy between various sites are not significant. The energy difference between the $\beta$ site and the over-hole $(\mathrm{OH})$ site, the least well bound of the binding sites, is only $0.05 \mathrm{eV}$ for $\mathrm{Ag}$ and $0.16 \mathrm{eV}$ for $\mathrm{Au}$. This indicates that the energy 


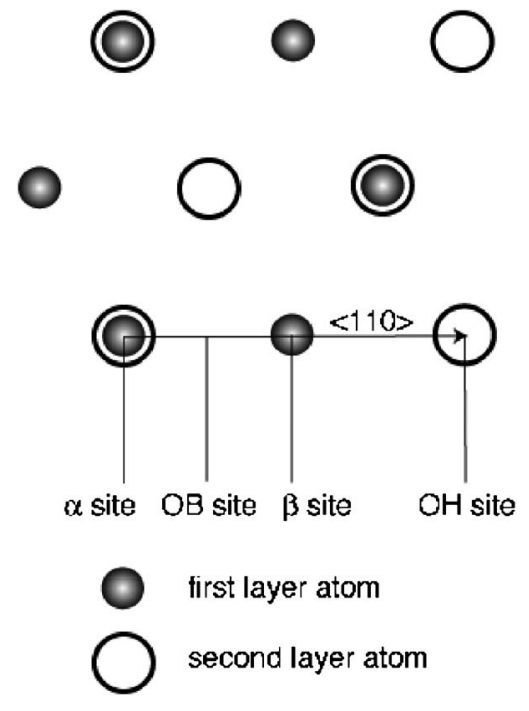

FIG. 1. Layout of graphite surface together with a description of the bonding sites considered for the adatom and dimer.

landscape for the adatoms on the graphite surface is very flat.

The methodology can also be used to determine the binding energies of larger clusters of $\mathrm{Ag}$ and $\mathrm{Au}$ atoms and examples of such results are also shown in Table I. The binding energies for the $\mathrm{Au}$ adatom and the dimer and the energy differences between the sites are larger than for the corresponding $\mathrm{Ag}$ values indicating that the gold adatoms and dimers are slightly less mobile than the silver ones. ${ }^{12,14}$ Nonetheless, assuming a typical attempt frequency of $10^{13}$ for surface processes, the bonding energy differences between the various sites along the $\langle 110\rangle$ direction would indicate a hop time of around $10^{-10} \mathrm{~s}$, for Au atoms so diffusion would be a fast process.

Even larger cluster interactions should be investigated for direct comparison to experiment and for fitting the $\mathrm{Ag}-\mathrm{C}$ interaction potential but such calculations are limited by current computing capabilities. Thus only the results from the interactions of the small clusters were used as a guide to fit a pairwise interaction potential to model the Ag-C and Au-C interactions. The details of this parametrization are given in the next section.

\section{MOLECULAR DYNAMICS METHODOLOGY}

The geometrical arrangement of a free cluster was found by applying a genetic algorithm ${ }^{15}$ to a family of random spatial configurations of atoms, interacting with each other through a many-body potential function parametrized by Ackland et al. ${ }^{16}$ This algorithm is capable of determining minima in structures containing several hundred atoms. The clusters of size seven and 55 atoms and are especially stable and exhibit icosahedral symmetry. The seven-atom cluster consists of five atoms arranged in a regular pentagonal structure with the other two atoms placed symmetrically above and below the pentagon. All faces of the polyhedron are equilateral triangles. This same potential was employed during the impact simulations.

The cluster was placed above the substrate outside the potential interaction range with internal kinetic energy before impact corresponding to a temperature of $300 \mathrm{~K}$. The substrate area was taken as $180 \AA \times 180 \AA$ with nine graphite layers containing 110376 atoms. This size was found to be sufficient in that increasing the numbers of atoms beyond this did not change the calculated pinning thresholds. The covalent $\mathrm{C}-\mathrm{C}$ interaction was modeled by a many-body Brenner $^{17,18}$ potential while an additional long-range Lennard-Jones potential ${ }^{19}$ between atoms that are not linked by covalent bonds was employed to take into account the Van der Waals interaction between graphite layers. The carbon atoms at the edges were fixed, and adjacent atoms undergo a damped force to prevent lattice displacement waves reflecting back into the impact zone. These waves are quite strong and can displace the surface layers by several A. They propagate in a hexagonal manner from the impact point. ${ }^{20}$ For each cluster size, the cluster energy was increased in steps of $25 \mathrm{eV}$ until a permanent defect in the substrate was observed. Only one cluster orientation and impact point was considered in generating the results since test calculations showed that the effect of impact point and orientation for large clusters affected the results by at most $\pm 25 \mathrm{eV}$. Because the calculations were only carried out in increments of $25 \mathrm{eV}$, a finer uncertainty cannot be determined without further calculations.

Both the $\mathrm{Ag}-\mathrm{C}$ and the $\mathrm{Au}-\mathrm{C}$ interactions were modeled in the system by a Morse potential. This potential was fitted so that the binding energy of atoms to the surface was as close as possible to that determined by the $a b$ initio calculations described in the previous section. Although another potential had already been used to model Ag clusters interacting with surfaces, ${ }^{3}$ it was found that the previous parametrization overestimated the binding energy of the $\mathrm{Ag}$ adatom and dimer by about a factor of 2 . Such an increase of the binding

TABLE I. Binding energies of the Ag and Au adatom and dimer with the graphite surface along [110]. The lowest energy dimer position is when the dimer axis is aligned near to the surface normal. The results for the Au trimer and tetramer are for vertically oriented planar clusters which are the lowest energy configurations with the exception of the $\alpha$ site for the Au trimer. In this case a configuration with the trimer parallel to the surface is $0.02 \mathrm{eV}$ more favorable at $1.34 \mathrm{eV}$ (Ref. 14).

\begin{tabular}{ccccccc}
\hline \hline Site & $\begin{array}{c}\text { Au atom } \\
(\mathrm{eV})\end{array}$ & $\begin{array}{c}\text { Au dimer } \\
(\mathrm{eV})\end{array}$ & $\begin{array}{c}\text { Au trimer } \\
(\mathrm{eV})\end{array}$ & $\begin{array}{c}\text { Au tetramer } \\
(\mathrm{eV})\end{array}$ & $\begin{array}{c}\text { Ag atom } \\
(\mathrm{eV})\end{array}$ & $\begin{array}{c}\text { Ag dimer } \\
(\mathrm{eV})\end{array}$ \\
\hline$\alpha$ & 0.662 & 1.129 & 1.32 & 1.28 & 0.430 & 0.513 \\
$\beta$ & 0.674 & 1.137 & 1.36 & 1.32 & 0.439 & 0.511 \\
bridge $(\mathrm{OB})$ & 0.654 & 1.154 & 1.36 & 1.32 & 0.434 & 0.523 \\
hollow $(\mathrm{OH})$ & 0.512 & 0.919 & 1.21 & 0.97 & 0.392 & 0.552 \\
\hline \hline
\end{tabular}


energy was found to reduce the pinning threshold slightly; as a result, the fitting constants were reevaluated.

The binding between the cluster atoms and the substrate was fitted using a pairwise Morse function of the form

$$
V(r)=D_{E}\left\{\exp \left[-2 \beta\left(r-r_{e}\right)\right]-2 \exp \left[-\beta\left(r-r_{e}\right)\right]\right\} .
$$

Here $r$ is the pair separation distance in $\AA$. The constants are given by $D_{E}=0.153 \mathrm{eV}$, for $\mathrm{Au}$ and $D_{E}=0.101 \mathrm{eV}$ for $\mathrm{Ag}$, $\beta=2.6$ for Au and 2.66171 for $\mathrm{Ag}$, and $r_{e}=2.6 \AA$ for $\mathrm{Au}$ and and $2.655 \AA$ for $\mathrm{Ag}$.

In addition, we splined the metal-C potential to the twobody Ziegler-Biersack-Littmark (ZBL) screened Coulomb potential ${ }^{21}$ for high interaction energies so that for small interatomic separation, $r<r_{a}$ the potential was entirely determined by the ZBL part. The ZBL potential has been shown to give good agreement with ab initio calculations and is fit to large quantitities of ion implantantion data. For $r_{a} \leqslant r$ $\leqslant r_{b}$ a cubic exponential splining function was used to make the potential and forces continuous at the joining points. For Au we used $r_{a}=1.2 \AA$ and $r_{b}=2.0 \AA$; for $\mathrm{Ag}$, we used $r_{a}$ $=1.8 \AA$ and $r_{b}=2.1 \AA$.

Although the parameters were chosen as closely as possible to fit the $a b$ initio binding energies, it is not possible to do this exactly with a pair potential and there are consequently some differences in the behavior. It was possible to match the optimum height above the surface for the adatom but for both Ag and Au the pair potential gave the over-hole site as the preferred minimum configuration rather than the $\beta$ site predicted by the ab initio calculations. The bridge site was also marginally preferred to the $\beta$ site.

The whole system was initially heated up by thermostat until equilibration at a temperature of $300 \mathrm{~K}$, but was switched off during the impact simulation to prevent the extraction of energy from the system by the thermostat. Normal incidence was always considered.

For the Ni-C interactions no ab initio calculations have been carried out and so the same pairwise Morse form for the bonding of the $\mathrm{Ni}$ atoms to the surface was chosen as for the $\mathrm{Au}$ atoms.

Since the atomic spacing for $\mathrm{Ag}$ and $\mathrm{Au}$ atoms are similar, the main differences between the computed results for $\mathrm{Ag}$ and $\mathrm{Au}$ should be due mostly to the different masses of the impacting clusters. The mass ratio between $\mathrm{Ni}$ and $\mathrm{Ag}$ is similar to that between $\mathrm{Ag}$ and $\mathrm{Au}$ so difference between the $\mathrm{Ni}$ and $\mathrm{Ag}$ results compared to the $\mathrm{Ag}$ and $\mathrm{Au}$ data should be due to the different cluster sizes. There was a small variation of the calculated pinning thresholds depending on impact site, cluster orientation, and temperature $(0-300 \mathrm{~K})$. As a result the calculated thresholds vary up to $50 \mathrm{eV}$ dependent on the precise conditions of impact.

A few simulations were also run with the new Ag potential in order to check that previous calculations with the old parametrization were accurate.

\section{RESULTS AND DISCUSSION}

The level of agreement between the experimental pinning thresholds obtained from scanning tunneling microscopy (STM) after deposition at different energies and those ob-

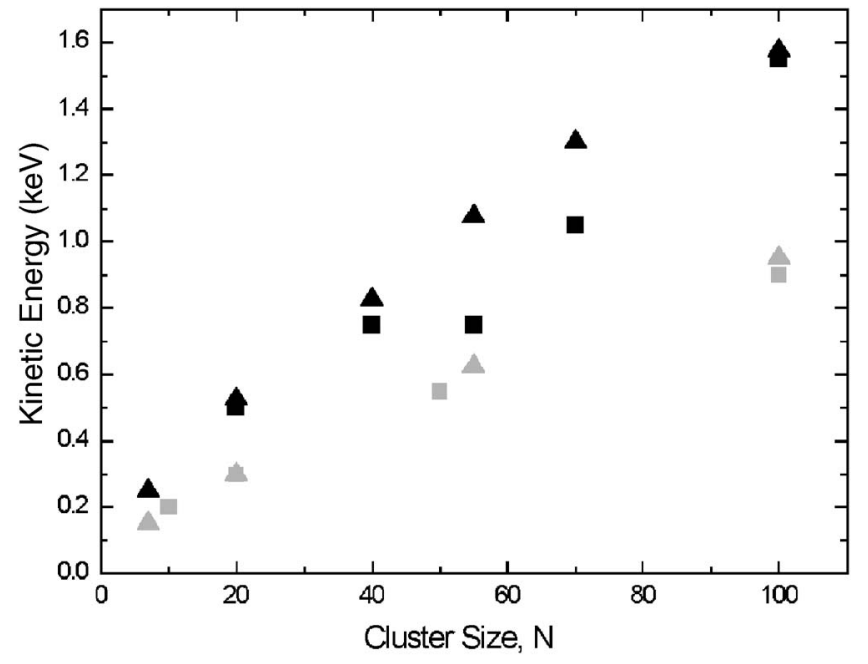

FIG. 2. The pinning energy thresholds for Au (dark symbols) and Ni (gray symbols) clusters. The square symbols are the experimental results and the triangles the MD. There is an uncertainty of about $\pm 25 \mathrm{eV}$ in the MD results dependent on the precise impact point and cluster orientation.

tained from the MD simulations, as shown in Fig. 2, is remarkably good overall. The only real exception is for the $\mathrm{Au}_{55}$ cluster. Generally there is also a slight overprediction of the value of $E_{P}$ compared to experiment. Although the curves are shown as passing through the origin, it is meaningless to consider very small clusters since for these, the impact point becomes important and it is then necessary to run a representative set of trajectories to determine average behavior. In fact pinning in the surface for small clusters from $N=1-$ $\approx 5$ becomes a less common occurrence than implantation beneath (if the energy is too large), reflection, or entrapment in the surface binding potential without pinning (for smaller energies).

The simulations also show a variety of detailed atomic processes leading to the formation of a surface defect and hence the pinning of the cluster. One mechanism already reported is when at least one $\mathrm{C}$ atom in surface layer under the cluster is knocked into an interstitial position between the first and second layers and replaced by a cluster atom. ${ }^{3} \mathrm{Al}-$ though this is a common mechanism, some other mechanisms were also observed. In some cases a first layer $\mathrm{C}$ atom could be knocked into an adatom position above the surface. In another example a cluster atom could become trapped between the first two graphite layers which themselves remained broadly intact. A final mechanism involves the formation of a "crease" in the surface where two or more surface $\mathrm{C}$ atoms begin to form bonds with corresponding second layer atoms while retaining their first layer neighbors. The length of the bond between atoms in the first layer and second layers is longer than that between those in the first layer at around $2 \AA$ so this appears to be an intermediate stage towards an $s p^{3}$ bonded configuration induced by the local momentum transfer to the substrate.

It is interesting to examine in detail the energy required to induce these processes. As a background, the formation energy of a vacancy, $E_{V F}$ has been determined experimentally 
as about $7.0 \mathrm{eV} .{ }^{22}$ Reported values of the interstitial formation energy are somewhat smaller, lying between 5.5 and $7.0 \mathrm{eV} .{ }^{23}$ However the amount of energy required to be imparted to a stationary $\mathrm{C}$ atom in a lattice at rest, to remove it permanently from its lattice site (the displacement energy threshold $E_{D}$ ), is $33 \mathrm{eV} .{ }^{19}$

In previous work with Ag clusters, a simple relationship for the pinning threshold based on a binary elastic collision model, treating the impacting cluster as a single object, has been proposed. If we assume that, at the moment of impact, we have a massive body (the cluster) colliding with a light body (the recoil carbon atoms that lie under the cluster), then from conservation of energy and momentum for an elastic collision, the energy transferred to the recoil carbon atoms, $E_{T r}$, is given by

$$
E_{T r} \approx 4 \frac{N_{C} M_{C} E_{P}}{N M_{C l u s}}
$$

where $M_{C}$ is the mass of the carbon atom, $N_{C}$ the number of carbon atoms set into motion by the impact, and $M_{\text {Clus }}$ the mass of the cluster atoms. If a certain value of $E_{T r}$ is to be surpassed before a $\mathrm{C}$ atom displaces, then $E_{P}$ can be determined. Previously it was assumed that only one $\mathrm{C}$ atom was set in motion, ${ }^{4,9}$ so that $N_{C}=1$ and then $E_{P} \propto N$. On the other hand if the cluster sets in motion all atoms in the first layer, that lie under the cluster, then $N_{C} \propto N^{2 / 3}$ and we would have $E_{P} \approx N^{1 / 3}$. We might therefore expect a dependence of $E_{P}$ with $N$ that was somewhere between these two extreme cases.

In practice, the data in Fig. 2 can be fitted fairly well with an offset linear plot. If the origin is included a $\log$ plot of $E_{P}$ against $N$ gives $E_{P} \propto N^{s}$, with $s \approx 0.6$, for both the Au and Ni data. This lies within the expected range of $\frac{1}{3}<s<1$. Both formulas predict that $E_{P} \propto M_{C l u s}$ when cluster atoms have the same atomic spacing. Since Au and Ag have approximately the same lattice constant, then proportionality should hold. Indeed if we consider the 55-atom cluster with the calculated values of $E_{P}$ of $600 \mathrm{eV}$ (Ref. 4) and $1075 \mathrm{eV}$ for $\mathrm{Ag}$ and $\mathrm{Au}$ respectively, the ratio of these threshold energies is 1.79 , whereas the mass ratio is 1.84 which agrees very well. However, the pinning thresholds for $\mathrm{Ni}$ are only slightly less than those for $\mathrm{Ag}$, despite the mass ratio being 1.83, almost the same as that between $\mathrm{Au}$ and $\mathrm{Ag}$. In this case the Ni lattice spacing is $14 \%$ smaller than for $\mathrm{Ag}$, so that for $\mathrm{Ni}$ clusters the energy density deposited in the surface is more concentrated and fewer surface atoms are set in motion. The optimization process shows that the $\mathrm{Ni}$ and $\mathrm{Au}$ clusters of equivalent size have the same morphology before impact. They also have similar cohesive energies so the differences between the values of $E_{P}$ are mainly due to the smaller lattice constant for $\mathrm{Ni}$ and the heavier mass of the Au cluster.

In order to determine the value of $E_{T r}$ from the simulations, the kinetic energy of the atoms in the graphite lattice was monitored so that a value for each atom could be determined. This shows that the maximum kinetic energy of substrate $\mathrm{C}$ atom occurs between 100 and 200 fs after the cluster impact with the surface. The maximum kinetic energy transferred to an atom was found to vary between 4.75 and

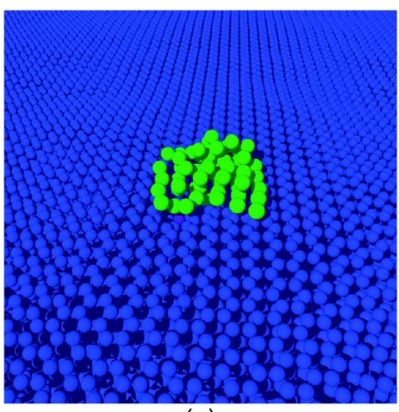

(a)

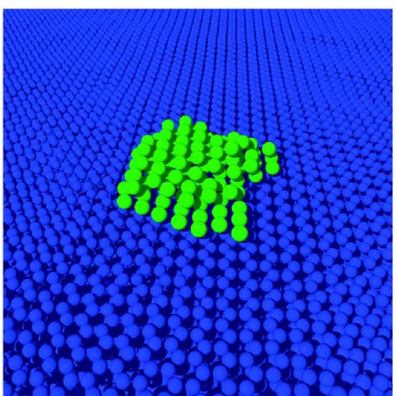

(c)

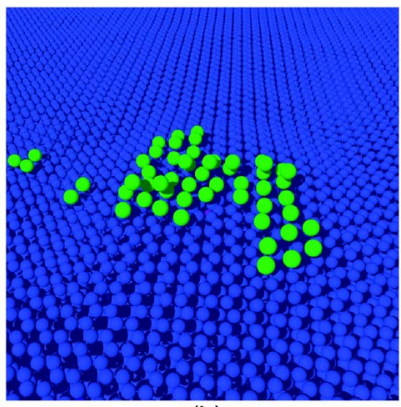

(b)

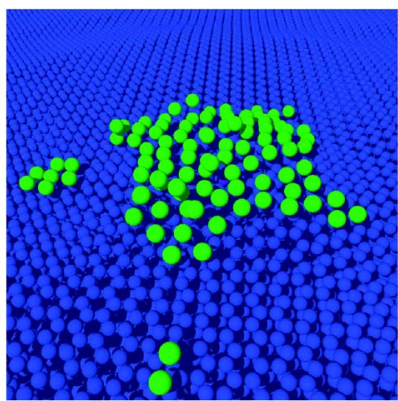

(d)
FIG. 3. (Color online) Images of the pinned clusters on the graphite surface; (a) 55 atom Ni cluster; (b) 55 atom Au cluster; (c) 100 atom Ni cluster; (d) 100 atom Au cluster.

$6.25 \mathrm{eV}$ irrespective of cluster size in the range $N=7-100$, but dependent on the impact position and orientation. These values are similar to the vacancy or interstitial formation energies reported above. However the molecular dynamics (MD) simulations also show that a number of atoms that lie under the cluster are set in motion simultaneously in a correlated effect. The result is that surface defects can form without an atom having to attain anything approaching the energy of $E_{D}$.

Turning now to the morphology of the deposited clusters, the geometry of clusters 10 ps after impact are shown in Fig. 3 . These simulations show that the Au clusters are more spread out after impact while the Ni clusters are more compact. In fact some fragmentation of the Au clusters takes place. The small fragments are not pinned but trapped in the surface binding potential and hence would be expected to diffuse quickly over the surface and recombine since as has already been pointed out, the barriers for diffusion are small.

Although some caution is required in interpreting height data from experiment due to tip convolution electronic and oxide effects, the heights of the clusters in the simulation are in general agreement with the ambient STM measurements obtained from sampling a range of clusters on the surface. ${ }^{9}$ The measurements show that the most commonly observed cluster height for the $\mathrm{Au}_{55}$ cluster at $1.15 \mathrm{keV}$ is that of a single adatom layer (see Fig. 4) whereas for a $\mathrm{Ni}_{250}$ cluster deposited at $1.8 \mathrm{keV}$ the most common height is three layers above the surface. The images shown in Fig. 3 confirm the general observation that $\mathrm{Ni}$ clusters are higher whereas $\mathrm{Au}$ clusters are spread out. Both the $\mathrm{Ni}_{55}$ and $\mathrm{Ni}_{100}$ clusters are quite compact with up to four layers of $\mathrm{Ni}$ atoms whereas there are only a few second layer adatoms for the corresponding Au clusters. 


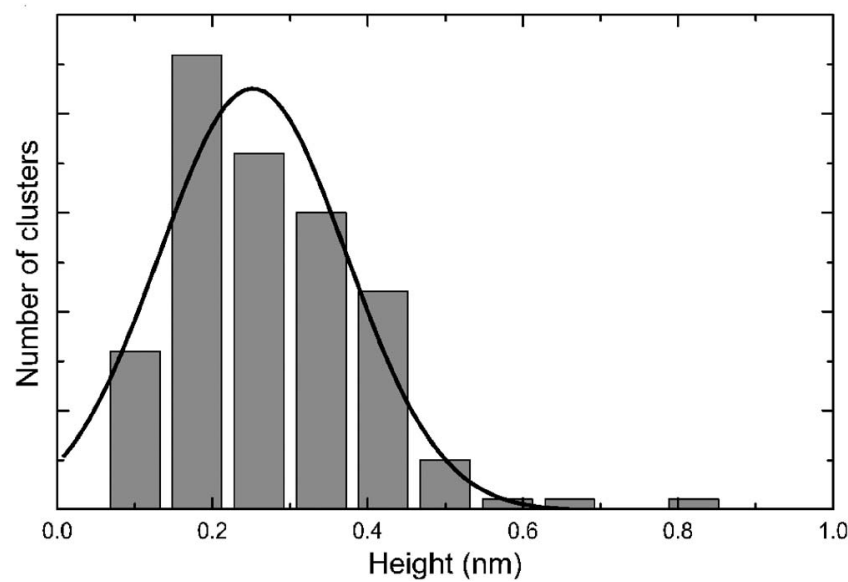

FIG. 4. The height distribution from experimentally measured $\mathrm{Au}_{55}$ clusters deposited on graphite at $1.15 \mathrm{keV}$. The total number of clusters analyzed is exactly 650 .

Calculations with the new parametrized Ag-C potential give similar results as before. ${ }^{4}$ Due to computing constraints the previous results were run with smaller systems. The reduced binding energy with the new potential results in a slight increase in the pinning threshold but this is compensated for by the larger substrate system in the current investigations which reduces the pinning threshold by more efficiently dispersing the energy in the surface waves produced by the impact and reducing the energy in any reflected waves.

\section{CONCLUSION}

Classical MD simulations have been carried out to investigate the pinning of size-selected $\mathrm{Au}$ and $\mathrm{Ni}$ clusters on the graphite surface. As for previous work with $\mathrm{Ag}$ clusters the pinning energy thresholds have been shown to be in good agreement with experiment. Pinning occurs by the formation of a surface defect and a number of different mechanisms for defect formation by the impact have been identified. In addition the height of the $\mathrm{Au}$ and $\mathrm{Ni}$ clusters on the surface show the same trends as the experimental data.

Previous work for $\mathrm{Ag}$ clusters found a variation of the pinning energy threshold with cluster size scaling with $N^{s}$, where $s \approx 1$. In the present case, the results can again be fitted by a linear relation if an offset is allowed. If the origin is included, the data is well represented with $s \approx 0.6$.

Comparison of different kinds of clusters indicates there is also a dependence of the pinning thresholds on the cluster mass with the heavier clusters requiring a larger energy to pin. The extra momentum required for the heavier clusters means that they are less compact after pinning and cover a bigger surface area than the lighter materials. This observation is in agreement with experiment STM measurements.

The modeling is also consistent with other experimental observations. The binding energy for small clusters, calculated by $a b$ initio methods, is very similar at different sites on the defect-free surface and it would be expected that the same would apply for larger clusters. Thus unpinned clusters would be highly mobile and diffuse to surface defects. Below the threshold for pinning, STM images of large areas of the graphite surface show no clusters.
${ }^{1}$ R. E. Palmer, S. Pratontep, and H.-G. Boyen, Nat. Mater. 2, 443 (2003).

${ }^{2}$ C. Leung, C. Xirouchaki, N. Berovic, and R. E. Palmer, Adv. Mater. (Weinheim, Ger.) 16, 223 (2004).

${ }^{3}$ S. J. Carroll, P. D. Nellist, R. E. Palmer, S. Hobday, and R. Smith, Phys. Rev. Lett. 84, 2654 (2000).

${ }^{4}$ S. J. Carroll, S. Pratontep, M. Streun, R. E. Palmer, S. Hobday, and R. Smith, J. Chem. Phys. 113, 7723 (2000).

${ }^{5}$ R. Smith, S. D. Kenny, C. F. Sanz-Navarro, and J. J. Belbruno, J. Phys.: Condens. Matter 15, S3153 (2003).

${ }^{6}$ S. Pratontep, P. Preece, C. Xirouchaki, R. E. Palmer, C. F. SanzNavarro, S. D. Kenny, and R. Smith, Phys. Rev. Lett. 90, 055503 (2003).

${ }^{7}$ D. J. Kenny, R. E. Palmer, C. F. Sanz-Navarro, and R. Smith, J. Phys.: Condens. Matter L185, 14 (2002).

${ }^{8}$ C. F. Sanz-Navarro, R. Smith, D. J. Kenny, S. Pratontep, and R. E. Palmer, Phys. Rev. B 65, 165420 (2002).

${ }^{9}$ M. Di Vece, S. Palomba, and R. E. Palmer, Phys. Rev. B 72, 073407 (2005).

${ }^{10}$ U. Prisco, C. Leung, C. Xirouchaki, C. H. Jones, J. K. Heath, and R. E. Palmer, J. R. Soc., Interface 2, 169 (2005).

${ }^{11}$ S. D. Kenny, A. P. Horsfield, and H. Fujitani, Phys. Rev. B 62,
4899 (2000)

${ }^{12}$ Guan Ming Wang, J. BelBruno, S. D. Kenny, and R. Smith, Surf. Sci. 541, 91 (2003).

${ }^{13}$ Guan Ming Wang, J. J. BelBruno, S. D. Kenny, and R. Smith, Phys. Rev. B 69, 195412 (2004).

${ }^{14}$ Guan Ming Wang, J. J. BelBruno, S. D. Kenny, and R. Smith, Surf. Sci. 576, 107 (2005).

${ }^{15}$ S. Hobday and R. Smith, J. Chem. Soc., Faraday Trans. 93, 3919 (1997).

${ }^{16}$ G. J. Ackland, G. Tichy, V. Vitek, and M. W. Finnis, Philos. Mag. A 56, 735 (1987).

${ }^{17}$ D. W. Brenner, Phys. Rev. B 42, 9458 (1990).

${ }^{18}$ D. W. Brenner, Phys. Rev. B 46, 1948 (1992).

${ }^{19}$ R. Smith and K. M. Beardmore, Thin Solid Films 272, 255 (1996).

${ }^{20}$ R. Smith and R. P. Webb, Proc. R. Soc. London, Ser. A 441, 495 (1993).

${ }^{21}$ J. F. Ziegler, J. P. Biersack, and U. Littmark, The Stopping and Range of Ions in Solids (Pergamon, New York, 1985).

${ }^{22}$ J. G. Kushmerick, K. F. Kelly, H. P. Rust, N. J. Halas, P. S. Weiss, J. Phys. Chem. B 103, 1619 (1999).

${ }^{23} \mathrm{M}$. Heggie (private communication). 